OPEN ACCESS

Edited by:

Christos Argyropoulos, University of New Mexico,

United States

Reviewed by:

Ashish Kataria,

University of New Mexico,

United States

Feng Yu,

Peking University First Hospital, China

${ }^{*}$ Correspondence:

Kiyonori lto

kiyonori.ito@gmail.com

tThese authors have contributed equally to this work

Specialty section

This article was submitted to Nephrology,

a section of the journal

Frontiers in Medicine

Received: 04 December 2019 Accepted: 20 April 2020 Published: 15 May 2020

Citation:

Ito K, Ookawara S, Hibino Y, Imai S,

Fueki M, Bandai Y, Yasuda $M$ Kamimura T, Kakuda H, Kiryu S, Wada N, Hamashima Y, Kobayashi T,

Shindo M, Sanayama H, Ohnishi Y,

Tabei K and Morishita Y (2020)

Skeletal Muscle Mass Index Is

Positively Associated With Bone Mineral Density in Hemodialysis

Patients. Front. Med. 7:187

doi: 10.3389/fmed.2020.00187

\section{Skeletal Muscle Mass Index Is Positively Associated With Bone Mineral Density in Hemodialysis Patients}

\author{
Kiyonori Ito ${ }^{1 * t}$, Susumu Ookawara ${ }^{1 \dagger}$, Yutaka Hibino ${ }^{2}$, Sojiro Imai ${ }^{3}$, Mariko Fueki ${ }^{3}$, \\ Yusaku Bandai ${ }^{3}$, Masatoshi Yasuda ${ }^{3}$, Tatsuya Kamimura ${ }^{3}$, Hideo Kakuda ${ }^{4}$, Satoshi Kiryu ${ }^{3}$, \\ Noriko Wada ${ }^{3}$, Yuri Hamashima ${ }^{5}$, Tadanao Kobayashi ${ }^{6}$, Mitsutoshi Shindo ${ }^{1}$, \\ Hidenori Sanayama ${ }^{7}$, Yasushi Ohnishi ${ }^{8}$, Kaoru Tabei $^{8}$ and Yoshiyuki Morishita ${ }^{1}$
}

${ }^{1}$ Division of Nephrology, Department of Integrated Medicine, Saitama Medical Center, Jichi Medical University, Saitama, Japan, ${ }^{2}$ Department of Orthopaedic Surgery, Minami-Uonuma City Hospital, Niigata, Japan, ${ }^{3}$ Department of Dialysis, Minami-Uonuma City Hospital, Niigata, Japan, ${ }^{4}$ Department of Radiology, Minami-Uonuma City Hospital, Niigata, Japan, ${ }^{5}$ Department of Population Health Science, Bristol Medical School, University of Bristol, Bristol, United Kingdom,

${ }^{6}$ Department of Internal Medicine, Yuzawa-Machi Health Medical Center, Niigata, Japan, ${ }^{7}$ Department of Neurology, Department of Integrated Medicine, Saitama Medical Center, Jichi Medical University, Saitama, Japan, ${ }^{8}$ Department of Internal Medicine, Minami-Uonuma City Hospital, Niigata, Japan

Background: Patients with chronic kidney disease (CKD) are at risk for bone loss and sarcopenia because of associated mineral and bone disorders (MBD), malnutrition, and chronic inflammation. Both osteoporosis and sarcopenia are associated with a poor prognosis; however, few studies have evaluated the relationship between muscle mass and bone mineral density (BMD) in hemodialysis (HD) patients. The present study examined the association between skeletal muscle mass index (SMI) and BMD in the lumbar spine and femoral neck in HD patients.

Methods: Fifty HD patients (mean age, $69 \pm 10$ years; mean HD duration, $9.0 \pm$ 8.8 years) in Minami-Uonuma City Hospital were evaluated. BMD was measured by dual-energy X-ray absorptiometry, and SMI was measured by bioelectrical impedance analysis (InBody ${ }^{T M}$ ) after HD. The factors affecting lumbar spine and femoral neck BMD were investigated, and multivariate analysis was performed.

Results: In simple linear regression analysis, the factors that significantly affected the lumbar spine BMD were sex, presence of hypertension, presence of diabetes mellitus, body mass index, triglyceride level, grip strength, and SMI; the factors that significantly affected the femoral neck BMD were sex, HD duration, serum creatinine level, tartrate-resistant acid phosphatase 5b level, undercarboxylated osteocalcin (ucOC) level, N-terminal propeptide of type I procollagen level, grip strength, and SMI. In multivariate analysis, SMI (standardized coefficient: 0.578) was the only independent factor that affected the lumbar spine BMD; the independent factors that affected the femoral neck BMD were SMI (standardized coefficient: 0.468), ucOC (standardized coefficient: -0.366) and sex (standardized coefficient: 0.231). 
Conclusion: SMI was independently associated with the BMD in the lumbar spine and femoral neck in HD patients. The preservation of skeletal muscle mass could be important to prevent $\mathrm{BMD}$ decrease in $\mathrm{HD}$ patients, in addition to the management of CKD-MBD.

Keywords: bone mineral density, skeletal muscle mass index, hemodialysis, sarcopenia, osteoporosis, osteosarcopenia

\section{INTRODUCTION}

Low bone mineral density (BMD) is common in patients with end-stage renal disease and chronic kidney disease (CKD), and hemodialysis (HD) increases the risk for bone loss and fractures. Low BMD is associated with the occurrence of new fractures, reduced quality of life, and increased mortality $(1,2)$. Thus, it is important to manage mineral and bone disorders (MBD) associated with CKD (CKD-MBD) (3). Additionally, HD patients commonly have decreased skeletal muscle mass and decreased muscle strength, known as sarcopenia (4). Both osteoporosis and sarcopenia are reportedly associated with poor mortality $(4,5)$. However, few studies have evaluated the association between BMD and skeletal muscle mass. Furthermore, some reports have shown that muscle mass is positively associated with $\operatorname{BMD}(6,7)$, while other studies have not found this positive association (8). Therefore, the relationship between BMD and muscle mass in HD patients remains unclear. The present study examined the association between skeletal muscle mass index (SMI) and BMD in the lumbar spine and femoral neck in HD patients.

\section{MATERIALS AND METHODS}

The present study included patients with end-stage renal disease treated with intermittent HD who were older than 20 years. Patients with implanted pacemakers were excluded, as the presence of pacemaker prevents body fluid composition measurement using bioelectrical impedance analysis (BIA).

Fifty HD patients were recruited ( 31 men, 19 women; mean age, $69 \pm 10$ years; mean HD duration, $9.0 \pm 8.8$ years). The patients' characteristics are summarized in Table 1 . The causes of chronic renal failure were diabetes mellitus (17 patients), chronic glomerulonephritis (19 patients), nephrosclerosis (3 patients), and other (11 patients). Each patient underwent maintenance $\mathrm{HD}$ three times per week for $3-5 \mathrm{~h}$ in each session. Informed consent for study inclusion was obtained from each patient. The study was approved by the Institutional Review Board of Minami-Uonuma City Hospital (H29-12), Japan, and conformed to the provisions of the Declaration of Helsinki (revised in Tokyo in 2004).

\section{Data Collection}

Baseline patient characteristics and other relevant data, including the primary disease of CKD, were collected from the medical records. Blood pressure and pulse rate were measured with patients in the supine position before the HD session at which each SMI measurement was taken. Body mass index (BMI) was calculated using the dry weight (DW). Blood samples were obtained from the arteriovenous fistula before HD.

\section{Measurement of SMI, BMD, and Grip Strength}

SMI was calculated using body composition measurements obtained with multi-frequency BIA (InBody S10; InBody Japan, Tokyo, Japan). A skeletal muscle mass evaluation was performed after the last HD session; that is, patients were measured under DW so that the weight was not affected by the amount of body fluid. Then, the SMI was calculated by normalizing the skeletal muscle mass for height $\left(\mathrm{kg} / \mathrm{m}^{2}\right)$.

Dual energy X-ray absorptiometry (Horizon A, HOLOGIC Japan, Tokyo, Japan) was used to evaluate the BMD in the lumbar spine (L2-L4) and femoral neck. To minimize variations in BMD measurements, a single radiologic technologist performed scanning and BMD data calculations for all HD patients.

Grip strength was measured by a grip strength meter (Smedley's Hand Dynamo Meter, Matsumiya Ika Seiki, Tokyo, Japan). Grip strength was measured twice for the hand without a dialysis shunt before the HD session. The mean of the two measurements was used as the grip strength for each patient.

\section{Statistics}

Data are expressed as mean \pm standard deviation. The paired Student's $t$-test was used to compare two parameters. Intergroup correlations were evaluated by Pearson's correlation coefficient and linear regression analysis. Multivariate linear regression analysis was performed using the parameters with statistically significant correlations in univariate analyses $(p<$ 0.05 ) to determine the independent factors that influenced the lumbar spine BMD and femoral neck BMD. All analyses were performed using SPSS Statistics version 19.0 (IBM, Armonk, NY, USA). Differences with a $p<0.05$ were considered statistically significant.

\section{RESULTS}

The clinical parameters of the study participants are shown in Table 1. The lumbar spine BMD in all included HD patients was $0.91 \pm 0.21 \mathrm{~g} / \mathrm{cm}^{2}$. The lumbar spine BMD was significantly greater in men $\left(0.96 \pm 0.21 \mathrm{~g} / \mathrm{cm}^{2}\right)$ than in women $(0.80 \pm 0.20$ $\left.\mathrm{g} / \mathrm{cm}^{2}, p<0.01\right)$. The female neck BMD in all included HD patients was $0.56 \pm 0.12 \mathrm{~g} / \mathrm{cm}^{2}$. The femoral neck BMD was significantly greater in men $\left(0.60 \pm 0.12 \mathrm{~g} / \mathrm{cm}^{2}\right)$ than in women $\left(0.49 \pm 0.07 \mathrm{~g} / \mathrm{cm}^{2}, p<0.01\right)$. In addition, the lumbar spine BMD was significantly greater than the femoral neck BMD $(p<$ $0.001)$. The SMI in all patients was $6.10 \pm 1.20 \mathrm{~kg} / \mathrm{m}^{2}$, and the grip strength was $23 \pm 8 \mathrm{~kg}$.

Table 2 shows the correlations between each BMD and the clinical parameters. Simple linear regression analysis showed 
TABLE 1 | Patient characteristics.

\begin{tabular}{|c|c|}
\hline Characteristics & $n=50$ \\
\hline Sex male, $n(\%)$ & $31(64)$ \\
\hline Age (years) & $69 \pm 10$ \\
\hline HD duration (year) & $9.0 \pm 8.8$ \\
\hline \multicolumn{2}{|l|}{ Primary disease, $n(\%)$} \\
\hline Diabetes mellitus & $17(34)$ \\
\hline Nephrosclerosis & $3(6)$ \\
\hline Chronic glomerulonephritis & $19(38)$ \\
\hline Others & $11(22)$ \\
\hline \multicolumn{2}{|l|}{ Past medical history, $\boldsymbol{n}(\%)$} \\
\hline Hypertension & $44(88)$ \\
\hline Diabetes mellitus & $20(40)$ \\
\hline Bone fracture & $9(18)$ \\
\hline Ischemic heart disease & $8(16)$ \\
\hline \multicolumn{2}{|l|}{ Medication, $n$ (\%) } \\
\hline Vitamin D & $34(68)$ \\
\hline$P$ binder & $42(84)$ \\
\hline Calcimimetics & $11(22)$ \\
\hline Body mass index $\left(\mathrm{kg} / \mathrm{m}^{2}\right)$ & $22.1 \pm 4.6$ \\
\hline Systolic BP (mmHg) & $150 \pm 23$ \\
\hline Diastolic BP (mmHg) & $78 \pm 12$ \\
\hline Heart rate (/min) & $69 \pm 12$ \\
\hline \multicolumn{2}{|l|}{ Laboratory findings } \\
\hline Albumin (g/dL) & $3.5 \pm 0.4$ \\
\hline Serum sodium (mEq/L) & $138 \pm 3$ \\
\hline Serum potassium (mEq/L) & $4.9 \pm 0.7$ \\
\hline Serum calcium, mg/dL & $8.4 \pm 0.6$ \\
\hline Serum phosphate (mg/dL) & $5.1 \pm 1.1$ \\
\hline BUN (mg/dL) & $60 \pm 13$ \\
\hline $\mathrm{Cr}(\mathrm{mg} / \mathrm{dL})$ & $10.1 \pm 1.1$ \\
\hline $\mathrm{Hb}(\mathrm{g} / \mathrm{dL})$ & $11.2 \pm 0.9$ \\
\hline Total cholesterol (mg/dL) & $156 \pm 41$ \\
\hline Triglyceride (mg/dL) & $109 \pm 68$ \\
\hline C-reactive protein (mg/dL) & $0.5 \pm 0.9$ \\
\hline Intact PTH (pg/mL) & $154 \pm 109$ \\
\hline ALP (U/L) & $245 \pm 101$ \\
\hline $\mathrm{BAP}(\mu \mathrm{g} / \mathrm{mL})$ & $16 \pm 13$ \\
\hline TRACP-5b (mU/dL) & $491 \pm 240$ \\
\hline ucOC (ng/mL) & $31 \pm 15$ \\
\hline Total P1NP (ng/mL) & $257 \pm 154$ \\
\hline \multicolumn{2}{|l|}{ Physical status and BMD } \\
\hline Grip strength (kg) & $23 \pm 8$ \\
\hline Skeletal muscle mass index $\left(\mathrm{kg} / \mathrm{m}^{2}\right)$ & $6.1 \pm 1.2$ \\
\hline Lumbar spine bone density $\left(\mathrm{g} / \mathrm{cm}^{2}\right)$ & $0.91 \pm 0.21$ \\
\hline Proxymal femur bone density $\left(\mathrm{g} / \mathrm{cm}^{2}\right)$ & $0.56 \pm 0.12$ \\
\hline
\end{tabular}

that the factors that significantly affected the lumbar spine BMD were sex, presence of hypertension, presence of diabetes mellitus, BMI, triglyceride level, grip strength, and SMI; the
TABLE 2 | Correlations between clinical parameters and respective BMD.

\begin{tabular}{|c|c|c|c|c|}
\hline & \multicolumn{2}{|c|}{ Lumbar spine } & \multicolumn{2}{|c|}{ Femoral neck } \\
\hline & $r$ & $p$-value & $r$ & $p$-value \\
\hline Sex male, $n(\%)$ & 0.356 & 0.011 & 0.416 & 0.003 \\
\hline Age (years) & -0.266 & 0.062 & -0.275 & 0.053 \\
\hline HD duration (year) & -0.231 & 0.106 & -0.311 & 0.027 \\
\hline \multicolumn{5}{|l|}{ Past medical history, $\boldsymbol{n}(\%)$} \\
\hline Hypertension & 0.283 & 0.046 & 0.178 & 0.216 \\
\hline Diabetes mellitus & 0.330 & 0.019 & 0.178 & 0.216 \\
\hline Bone fracture & -0.227 & 0.112 & -0.258 & 0.078 \\
\hline Ischemic heart disease & -0.147 & 0.308 & -0.005 & 0.970 \\
\hline Body mass index $\left(\mathrm{kg} / \mathrm{m}^{2}\right)$ & 0.435 & 0.002 & 0.270 & 0.058 \\
\hline Systolic BP (mmHg) & 0.051 & 0.725 & -0.078 & 0.591 \\
\hline Diastolic BP (mmHg) & -0.178 & 0.217 & -0.195 & 0.175 \\
\hline Heart rate (/min) & -0.105 & 0.486 & -0.172 & 0.232 \\
\hline Albumin (g/dL) & 0.037 & 0.800 & 0.046 & 0.750 \\
\hline Serum sodium (mEq/L) & -0.065 & 0.652 & -0.261 & 0.167 \\
\hline Serum potassium (mEq/L) & -0.199 & 0.166 & 0.018 & 0.899 \\
\hline Serum calcium (mg/dL) & 0.111 & 0.443 & 0.061 & 0.675 \\
\hline Serum phosphate (mg/dL) & 0.054 & 0.707 & 0.107 & 0.461 \\
\hline BUN (mg/dL) & -0.052 & 0.718 & 0.072 & 0.620 \\
\hline $\mathrm{Cr}(\mathrm{mg} / \mathrm{dL})$ & 0.227 & 0.113 & 0.301 & 0.034 \\
\hline $\mathrm{Hb}(\mathrm{g} / \mathrm{dL})$ & -0.011 & 0.939 & -0.036 & 0.802 \\
\hline Total cholesterol (mg/dL) & 0.096 & 0.508 & 0.111 & 0.443 \\
\hline Triglyceride (mg/dL) & 0.298 & 0.035 & 0.278 & 0.051 \\
\hline C-reactive protein (mg/dL) & 0.036 & 0.801 & -0.005 & 0.972 \\
\hline intact PTH (pg/mL) & -0.017 & 0.905 & -0.182 & 0.205 \\
\hline ALP (U/L) & -0.075 & 0.606 & -0.209 & 0.146 \\
\hline $\mathrm{BAP}(\mu \mathrm{g} / \mathrm{mL})$ & -0.210 & 0.143 & -0.233 & 0.103 \\
\hline TRACP-5b (mU/dL) & -0.199 & 0.180 & -0.419 & 0.002 \\
\hline ucOC (ng/mL) & -0.175 & 0.223 & -0.382 & 0.006 \\
\hline total P1NP (ng/mL) & -0.145 & 0.314 & -0.380 & 0.006 \\
\hline Grip strength (kg) & 0.471 & 0.001 & 0.507 & $<0.001$ \\
\hline Skeletal muscle mass index $\left(\mathrm{kg} / \mathrm{m}^{2}\right)$ & 0.578 & $<0.001$ & 0.560 & $<0.001$ \\
\hline
\end{tabular}

$H D$, hemodialysis; $B P$, blood pressure; BUN, blood urea nitrogen; $C r$, creatinine; $H b$, hemoglobin; PTH, parathyroid hormone; ALP, alkaline phosphatase; BAP, bone specific alkaline phosphatase; TRACP-5b, tartrate-resistant acid phosphatase $5 b$; ucOC, undercarboxylated osteocalcin; P1NP, N-terminal propeptide of type I procollagen; BMD, bone mineral density.

factors that significantly affected the femoral neck BMD were sex, HD duration, serum creatinine level, tartrate-resistant acid phosphatase 5b level, undercarboxylated osteocalcin (ucOC) level, total N-terminal propeptide of type I procollagen level, grip strength, and SMI. Figure 1 shows that SMI was positively correlated with both the lumbar spine BMD $(r=0.578, p<$ $0.001)$ and the femoral neck BMD $(r=0.560, p<0.001)$. Additionally, the lumbar spine BMD was positively correlated with femoral neck BMD $(r=0.586, p<0.001$; Figure 1C).

In multivariate analysis, the only factor that significantly affected the lumbar spine BMD was the SMI (standardized coefficient: 0.578 ), while the femoral neck BMD was significantly affected by the SMI (standardized coefficient: 0.468), ucOC 

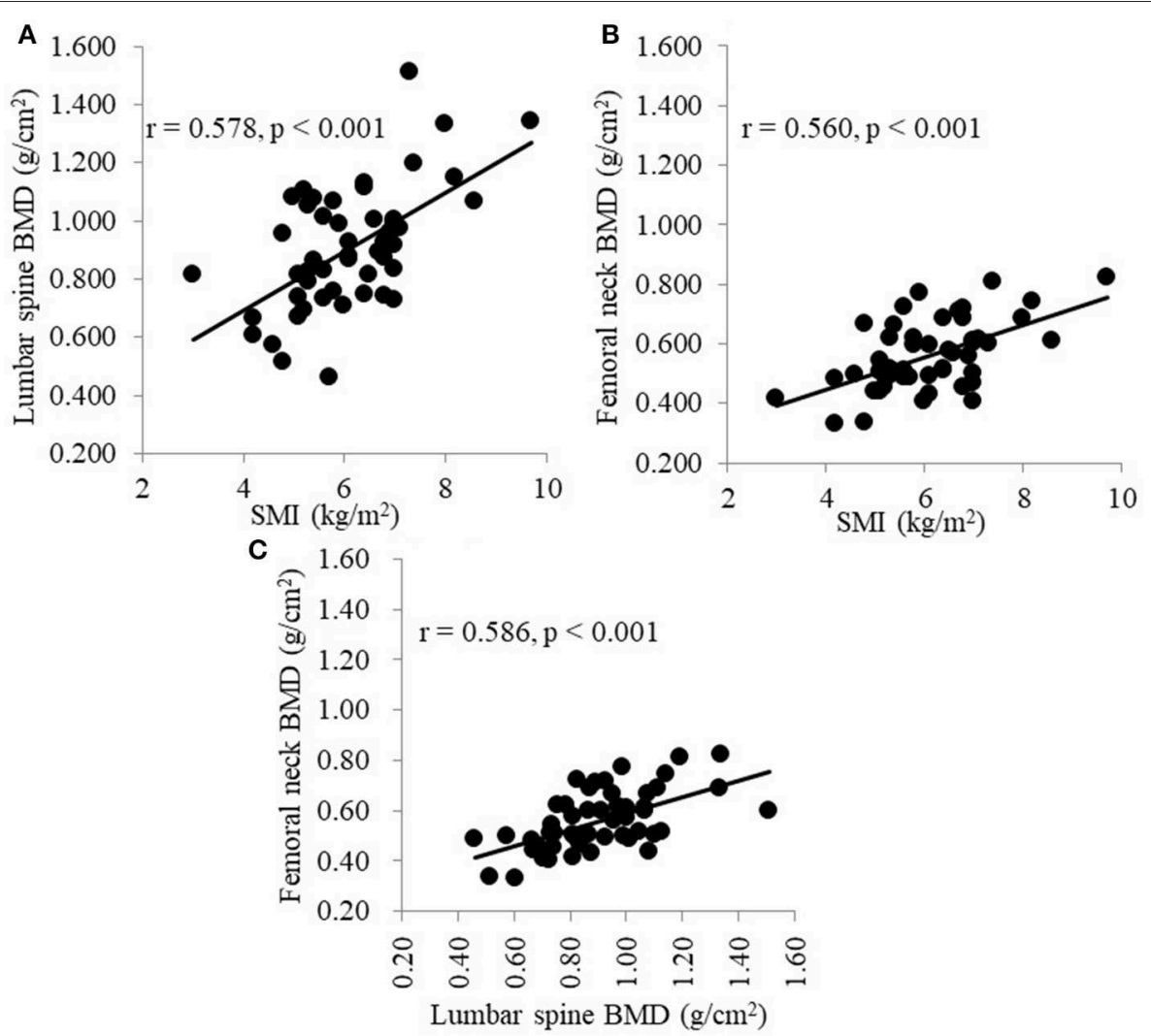

FIGURE 1 | (A) Correlation between SMI and the BMD of the lumbar spine. (B) Correlation between SMl and the BMD of the femoral neck. (C) Correlation between the BMD of the lumbar spine and the BMD of the femoral neck. SMI, skeletal muscle mass index; BMD, bone mineral density.

TABLE 3 | Multivariate regression analysis: Independent factors of BMD in the lumbar spine and femoral neck.

\begin{tabular}{llccr}
\hline BMD & Variables & Coefficient & $\begin{array}{c}\text { Standardized } \\
\text { coefficient }\end{array}$ & $\boldsymbol{p}$-value \\
\hline Lumbar spine & SMl & 0.101 & 0.578 & $<0.001$ \\
Femoral neck & SMl & 0.046 & 0.468 & $<0.001$ \\
& UCOC & -0.003 & -0.366 & 0.001 \\
& Sex (male:1) & 0.055 & 0.231 & 0.046 \\
\hline
\end{tabular}

$B M D$, bone mineral density; SMI, skeletal muscle mass index; ucOC, undercarboxylated osteocalcin.

level (standardized coefficient: -0.366 ), and sex (standardized coefficient: 0.231; Table 3).

\section{DISCUSSION}

The present study investigated the association between SMI and the BMD of the lumbar spine and the femoral neck. It is wellknown that the risk of bone fracture is increased in dialysis patients $(1,9)$, and malnutrition and sarcopenia are associated with poor prognosis $(10,11)$. However, reports regarding the association between osteoporosis and sarcopenia in HD patients are limited $(6,7,12)$. Similarly to these previous reports, our results demonstrated a positive correlation between SMI and
BMD. Muscle, especially skeletal muscle, supports the bones and positively supports physical activity (13). Therefore, it is important to understand osteosarcopenia in HD patients with both CKD-MBD and malnutrition.

Osteosarcopenia is a new concept that includes both osteoporosis and sarcopenia, and leads to an increased risk of frailty (14). The incidence of osteosarcopenia in general population is reportedly $5.8 \%$ (15) and this incidence is likely to be even higher in $\mathrm{HD}$ patients. The prevalence of osteosarcopenia in patients with $\mathrm{CKD}$ who have undergone kidney transplantation is reportedly $17.2 \%$, and these patients have a slower walking speed than patients without osteosarcopenia (16). In contrast, exercise contributes to BMD preservation (17), and exercise training improves grip strength and walking speed in dialysis patients (18). Therefore, the presence of osteosarcopenia, which decreases the ability to exercise, might be associated with negative cycles involving bone, skeletal muscle, and physical activity. In the clinical setting, HD patients must be properly managed, including the management of CKD-MBD to prevent both osteoporosis and sarcopenia.

In the present study, there were no correlation found between BMD and the levels of serum calcium, serum phosphate, and intact parathyroid hormone. Furthermore, BMD was not associated with bone turnover or osteogenesis markers. However, various therapeutic agents were used to appropriately manage the CKD-MBD status of patients undergoing HD and the CKD-MBD 
related data was well controlled. This might be the reason that these makers were not found to be significant independent factors associated with BMD in the present study. In contrast, previous studies have found that some bone turnover or osteogenesis markers were independently associated with $\operatorname{BMD}(6,7)$. These markers were also associated with the femoral neck BMD in simple liner regression performed in the present study. However, although bone metabolism-related makers were not associated with BMD, SMI was independently associated with BMD. A previous study reported that the femoral neck BMD was affected by body weight and serum creatinine level, which could reflect muscle mass (19). Therefore, the maintenance of skeletal muscle might be important in preventing BMD. Further prospective studies are required to clarify the association between CKD-MBD or bone metabolism and BMD.

In the present study, the femoral neck BMD was significantly affected by the ucOC level. Osteocalcin is one of the factors related to bone formation, and is induced by vitamin $K$. In patients with vitamin $\mathrm{K}$ deficiency, osteocalcin is mainly present in the circulation as ucOC, which is reportedly inversely correlated with vitamin K (20). Furthermore, an increased ucOC level is a risk factor for hip fracture (21), and vitamin K levels are also associated with bone fracture in HD patients (22). Therefore, improving vitamin $\mathrm{K}$ deficiency could be important for the transformation of osteocalcin. However, HD patients should pay attention to their diet, even if they are vitamin $\mathrm{K}$ deficient, as foods such as vegetables, tea, and seaweed that contain vitamin $\mathrm{K}$ have high levels of potassium. Although vitamin $\mathrm{K}$ and ucOC are not routinely measured in dialysis clinics, these might be useful measurements for maintaining BMD or preventing bone fracture.

Although grip strength had a strong correlation with BMD in simple linear regression in the present study, it was not found to be an independent factor affecting the BMD of the lumbar spine or femoral neck because grip strength is reportedly a good predictor of overall muscle strength and a useful marker of frailty (23). Furthermore, the distal radius BMD is reportedly one of the determinants of handgrip strength (7). If the present study has evaluated the muscle strength in the lumbar spine or femur, muscle strength might have been found to be an independent factor affecting the BMD. Therefore, the association between muscle strength and BMD should be investigated in future studies.

The present study has some limitations. First, the sample size was relatively small. Second, this study was a crosssectional study, and therefore, causal relationships cannot be

\section{REFERENCES}

1. Iimori S, Mori Y, Akita W, Kuyama T, Takada S, Asai T, et al. Diagnostic usefulness of bone mineral density and biochemical markers of bone turnover in predicting fracture in CKD stage 5D patients-a single-center cohort study. Nephrol Dial Transplant. (2012) 27:345-51. doi: 10.1093/ndt/gfr317

2. Tentori F, McCullough K, Kilpatrick RD, Bradbury BD, Robinson BM, Kerr PG, et al. High rates of death and hospitalization follow bone fracture among hemodialysis patients. Kidney Int. (2014) 85:166-73. doi: 10.1038/ki.2013.279 confirmed. Third, as HD patients are at increased risk of bone fracture or mortality due to the influences of CKD-MBD, various medications used to treat $\mathrm{CKD}-\mathrm{MBD}$ were prescribed throughout HD therapy. Therefore, a prospective longitudinal study is needed to investigate the associations between BMD and changes in CKD-MBD factors.

In conclusion, SMI was independently associated with the BMD of lumbar spine and femoral neck. Therefore, to prevent BMD decrease in HD patients, it may be important to preserve skeletal muscle mass in addition to performing CKDMBD management.

\section{DATA AVAILABILITY STATEMENT}

The datasets generated for this study are available on request to the corresponding author.

\section{ETHICS STATEMENT}

The studies involving human participants were reviewed and approved by the Institutional Review Board of Minami-Uonuma City Hospital (H29-12). The patients/participants provided their written informed consent to participate in this study.

\section{AUTHOR CONTRIBUTIONS}

KI conceived, designed, and performed the study, analyzed the data, and contributed to the writing of the manuscript. SO designed the study, analyzed the data, and contributed to the writing of the manuscript. YHi designed and performed the study, and supervised the writing of the manuscript. SI, MF, YB, MY, TKa, HK, SK, NW, YHa, TKo, and MS performed the study. $\mathrm{HS}$, YO, KT, and YM supervised the writing of the manuscript. All authors reviewed the manuscript.

\section{FUNDING}

This work was supported by a grant from The Kidney Foundation, Japan (JKFB 17-27).

\section{ACKNOWLEDGMENTS}

We thank the study participants, dialysis staffs, and radiology technologists at Minami-Uonuma City Hospital. We also thank Kelly Zammit, BVSc, from Edanz Editing (www.edanzediting. com/ac), for editing a draft of this manuscript. 
in end-stage renal disease patients: A cohort study. Bone. (2020) 130:115075. doi: 10.1016/j.bone.2019.115075

6. Tominaga H, Oku M, Arishima Y, Ikeda T, Ishidou Y, Nagano S, et al. Association between bone mineral density, muscle volume, walking ability, and geriatric nutritional risk index in hemodialysis patients. Asia Pac J Clin Nutr. (2018) 27:1062-6. doi: 10.6133/apjen.052018.03

7. Chen SC, Chung WS, Wu PY, Huang JC, Chiu YW, Chang JM, et al. Associations among Geriatric Nutrition Risk Index, bone mineral density, body composition and handgrip strength in patients receiving hemodialysis. Nutrition. (2019) 65:6-12. doi: 10.1016/j.nut.2019.02.013

8. Grzegorzewska AE, Młot-Michalska M. Total body mass is better than body mass index as a prognostic parameter for bone mineral density in dialyzed patients. Adv Perit Dial. (2009) 25:178-80. Available online at: https://www. advancesinpd.com/

9. Alem AM, Sherrard DJ, Gillen DL, Weiss NS, Beresford SA, Heckbert $\mathrm{SR}$, et al. Increased risk of hip fracture among patients with end-stage renal disease. Kidney Int. (2000) 58:396-9. doi: 10.1046/j.1523-1755.2000. 00178.x

10. Kanazawa Y, Nakao T, Murai S, Okada T, Matsumoto H. Diagnosis and prevalence of protein-energy wasting and its association with mortality in Japanese haemodialysis patients. Nephrology (Carlton). (2017) 22:5417. doi: $10.1111 /$ nep. 12814

11. Mori K, Nishide K, Okuno S, Shoji T, Emoto M, Tsuda A, et al. Impact of diabetes on sarcopenia and mortality in patients undergoing hemodialysis. BMC Nephrol. (2019) 20:105. doi: 10.1186/s12882-019-1271-8

12. Marinho SM, Wahrlich V, Mafra D. Association between body composition and bone mineral density in men on hemodialysis. Am J Med Sci. (2015) 350:286-9. doi: 10.1097/MAJ.0000000000000553

13. Morishita Y, Kubo K, Miki A, Ishibashi K, Kusano E, Nagata D. Positive association of vigorous and moderate physical activity volumes with skeletal muscle mass but not bone density or metabolism markers in hemodialysis patients. Int Urol Nephrol. (2014) 46:633-9. doi: 10.1007/s11255-0140662-9

14. Yoshimura N, Muraki S, Oka H, Iidaka T, Kodama R, Horii C, et al. Do sarcopenia and/or osteoporosis increase the risk of frailty? A 4-year observation of the second and third ROAD study surveys. Osteoporos Int. (2018) 29:2181-90. doi: 10.1007/s00198-018-4596-4

15. Yoshimura N, Muraki S, Oka H, Iidaka T, Kodama R, Kawaguchi H, et al. Is osteoporosis a predictor for future sarcopenia or vice versa? Four-year observations between the second and third ROAD study surveys. Osteoporos Int. (2017) 28:189-99. doi: 10.1007/s00198-016-3823-0
16. Yanishi M, Kinoshita H, Tsukaguchi H, Kimura Y, Koito Y, Sugi M, et al. Factors related to osteosarcopenia in kidney transplant recipients. Transplant Proc. (2018) 50:3371-5. doi: 10.1016/j.transproceed.2018.04.032

17. Polidoulis I, Beyene J, Cheung AM. The effect of exercise on pQCT parameters of bone structure and strength in postmenopausal women-a systematic review and meta-analysis of randomized controlled trials. Osteoporos Int. (2012) 23:39-51. doi: 10.1007/s00198-011-1734-7

18. Heiwe S, Jacobson SH. Exercise training in adults with CKD: a systematic review and meta-analysis. Am J Kidney Dis. (2014) 64:383-93. doi: 10.1053/j.ajkd.2014.03.020

19. Nakanishi K, Shishido K, Kumata C, Ito K, Nakashima Y, Wakasa M. Bone density of the femoral neck in patients on maintenance dialysis. PLoS One. (2018) 13:e0197965. doi: 10.1371/journal.pone.0197965

20. Miki T, Nakatsuka K, Naka H, Kitatani K, Saito S, Masaki H, et al. Vitamin $\mathrm{K}_{2}$ (menaquinone 4) reduces serum undercarboxylated osteocalcin level as early as 2 weeks in elderly women with established osteoporosis. J Bone Miner Metab. (2003) 21:161-5. doi: 10.1007/s007740300025

21. Vergnaud P, Garnero P, Meunier PJ, Bréart G, Kamihagi K, Delmas PD. Undercarboxylated osteocalcin measured with a specific immunoassay predicts hip fracture in elderly women: the EPIDOS Study. J Clin Endcrinol Metb. (1997) 82:719-24. doi: 10.1210/jc.82.3.719

22. Kohlmeier M, Saupe J, Shearer MJ, Schaefer K, Asmus G. Bone health of adult hemodialysis patients is related to vitamin K status. Kidney Int. (1997) 51:1218-21. doi: 10.1038/ki.1997.166

23. Purser JL, Kuchibhatla MN, Fillenbaum GG, Harding T, Peterson ED, Alexander KP. Identifying frailty in hospitalized older adults with significant coronary artery disease. J Am Geriatr Soc. (2006) 54:1674-81. doi: 10.1111/j.1532-5415.2006.00914.x

Conflict of Interest: The authors declare that the research was conducted in the absence of any commercial or financial relationships that could be construed as a potential conflict of interest.

Copyright (c) 2020 Ito, Ookawara, Hibino, Imai, Fueki, Bandai, Yasuda, Kamimura Kakuda, Kiryu, Wada, Hamashima, Kobayashi, Shindo, Sanayama, Ohnishi, Tabe and Morishita. This is an open-access article distributed under the terms of the Creative Commons Attribution License (CC BY). The use, distribution or reproduction in other forums is permitted, provided the original author(s) and the copyright owner(s) are credited and that the original publication in this journal is cited, in accordance with accepted academic practice. No use, distribution or reproduction is permitted which does not comply with these terms. 